\title{
Measurement of Hepatitis B Surface Antigen Concentrations Using a Piezoelectric Microcantilever as a Mass Sensor
}

\author{
Sangkyu Lee, ${ }^{1}$ Jongyun Cho, ${ }^{1}$ Yeolho Lee, ${ }^{2}$ Sangmin Jeon, ${ }^{3}$ \\ Hyung Joon Cha, ${ }^{3}$ and Wonkyu Moon ${ }^{1}$ \\ ${ }^{1}$ Department of Mechanical Engineering, Pohang University of Science and Technology, Pohang 790-784, Republic of Korea \\ ${ }^{2}$ Corporate Technology Operations SAIT, Samsung Electronics Co., Ltd., Yongin 446-712, Republic of Korea \\ ${ }^{3}$ Department of Chemical Engineering, Pohang University of Science and Technology, Pohang 790-784, Republic of Korea
}

Correspondence should be addressed to Wonkyu Moon,wkmoon@postech.ac.kr

Received 15 June 2011; Accepted 28 July 2011

Academic Editor: Martin Hegner

Copyright (c) 2012 Sangkyu Lee et al. This is an open access article distributed under the Creative Commons Attribution License, which permits unrestricted use, distribution, and reproduction in any medium, provided the original work is properly cited.

\begin{abstract}
Hepatitis B surface antigen (HBsAg) concentrations were measured using a piezoelectric microcantilever sensor (PEMS) developed by the authors. The developed PEMS is label-free and detects the sensing signal electrically. It was designed to measure the mass of biomolecules attached to it using an accurate mass-microbalancing technique; its probe area is confined to the end of the cantilever, and its equivalent spring constant is relatively high to minimize the effect of changes in the surface stress when the biomolecules are attached to it. The "dip- and-dry" technique was used to enable the probe area of the sensor to react with reagents in controlled environmental conditions. HBsAg was detected by an immunoreaction whereas the reaction time, antibody density, and its area on the probe were kept at a constant level. The mass of the detected HBsAg was measured in the range of $0.1-100 \mathrm{ng} / \mathrm{mL}$.
\end{abstract}

\section{Introduction}

Hepatitis B virus (HBV) infection causes the disease hepatitis $B$ and may also lead to cirrhosis and hepatocellular carcinoma [1]. An estimated two billion people worldwide have been infected by HBV, and of these, 350 million are chronically infected. Hepatitis B surface antigen ( $\mathrm{HBsAg}$ ) forms part of the surface of the virus and is used as a biomarker for the HBV infection [1]. We need to detect HBsAg in very low concentrations to accurately diagnose HBV. In hospitals, chemiluminescence immunoassay is widely used to detect $\mathrm{HBsAg}$, and its detection limit is approximately $0.05 \mathrm{ng} / \mathrm{mL}$ [2].

A piezoelectric microcantilever sensor (PEMS) offers many advantages as a biosensor and is suitable for the detection of HBsAg. A PEMS is a highly sensitive label-free sensor that is sufficiently small to be developed as a portable device; multiplexed detection and electrical readout are also available [3]. Many studies have been conducted using a PEMS to detect various biomarkers [4].

The principle of detection in a PEMS is based on changes in the resonant frequency of the PEMS before and after a target protein is attached to it; target proteins are captured on the probe area of the PEMS by an immunoreaction. The frequency changes depend on changes in the surface stress and mass loading due to the attached biomolecules. The influence of the surface stress on the resonant frequency decreases as the effective stiffness (spring constant) of the PEMS increases $[5,6]$. If we use a PEMS as a mass sensor based on a mass-microbalancing technique [7], the effective stiffness should be sufficiently large, and then, the resonant frequency will vary only in response to the mass loading effects.

The experimental setup consists of a part that measures the resonant frequency and a part that enables the probe area of the sensor to react with reagents. An impedance analyzer is usually used to measure the resonant frequency of the PEMS by detecting the peak point of the phase angle [8], dielectric loss [6], and so forth. The "dip- and-dry" technique is widely used in detection experiments [9]. The PEMS is dipped into reagents to either immobilize the antibody or bind the antigen, and the resonance frequencies are detected in air. The quality factor of the PEMS should be large because it is related to the accuracy of the detection of the resonant 
frequency change. A number of studies in the literature have reported the detection of biomolecules with a PEMS that is operated in a liquid, but the quality factor is greatly reduced in liquids [10]. Environmental conditions such as humidity and temperature must be controlled at a constant level because the resonance frequency of a PEMS can be affected by these conditions.

In this study, we demonstrate the measurement of HBsAg concentrations with a PEMS we developed that functions as a mass sensor. In order for the PEMS to function as a mass sensor, it was designed to have relatively large effective stiffness and the probe area was confined to the end of the device. The "dip- and-dry" technique was used, and the masses of detected HBsAg were measured in different concentrations and with different reaction times. Moreover, a control test using other proteins was performed.

\section{Piezoelectric Microcantilever as Mass Sensor}

The developed PEMS was previously designed by the authors to have sufficient sensitivity and reliability as a mass sensor $[7,11]$; the geometrical shape and dimensions of the sensor are shown in Figure 1(a). The piezoelectric layer is composed of lead zirconate titanate (PZT), whose composition is $\mathrm{Pb}\left(\mathrm{Zr}_{52} \mathrm{Ti}_{48}\right) \mathrm{O}_{3}$, and the cantilever structure is made of silicon. Gold is patterned on the end of the cantilever in the probe area.

A mechanical lumped parameter model, which is shown in Figure 1(b), is used to understand the mechanical characteristics of the PEMS. The lumped parameters are calculated from the modal analysis results of the PEMS using a commercial finite element analysis tool, COMSOL Multiphysics software. The material properties and the calculated value of the lumped parameters are listed in Table 1.

The mass sensitivity of the developed PEMS is over a million times higher than that of a commercial quartz crystal microbalance (QCM). The mass sensitivity of the PEMS is approximately $175 \mathrm{~Hz} / \mathrm{pg}$, which is calculated from an eigenvalue analysis before and after the addition of mass on the probe area (Figure 1(a)), and the mass sensitivity of the widely used QCM with a $5 \mathrm{MHz}$ crystal is approximately $79 \mathrm{~Hz} / \mu \mathrm{g}$ [13]. On the other hand, both sensors are comparable in the mass sensitivity per area because the probe area of the QCM $\left(\sim 1.267 \mathrm{~cm}^{2}\right)$ is over a million times larger than that of the PEMS $\left(\sim 50 \mu \mathrm{m}^{2}\right)$. The mass sensitivity per area of the PEMS is $87.5 \mathrm{~Hz} /\left(\mu \mathrm{g} / \mathrm{cm}^{2}\right)$ and that of the QCM is $100 \mathrm{~Hz} /\left(\mu \mathrm{g} / \mathrm{cm}^{2}\right)$.

Because the surface stress effect on the resonant frequency of the developed PEMS is quite small, the PEMS can be used as a mass sensor. If we assume that the initial surface stress $s$ is zero and that the probe area covers the entire area on one side of the rectangular-shaped microcantilever, the surface stress sensitivity is shown as follows [5]:

$$
\frac{1}{f_{0}} \frac{d f}{d s}=\frac{3}{16} \frac{1}{K_{\mathrm{eff}}} .
$$

The surface stress sensitivity is inversely proportional to the effective stiffness. If the probe area is reduced to one-tenth

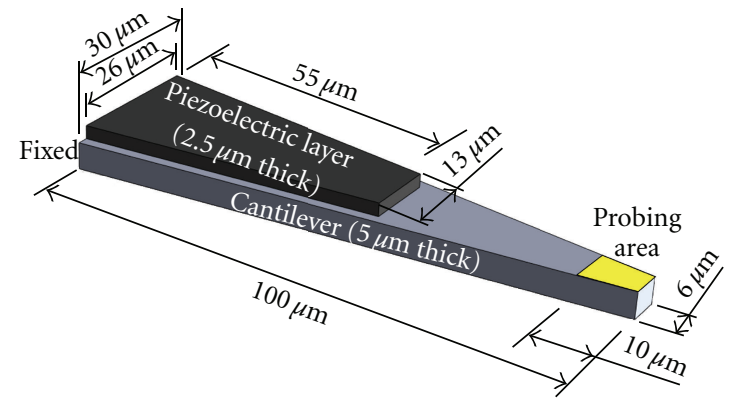

(a)

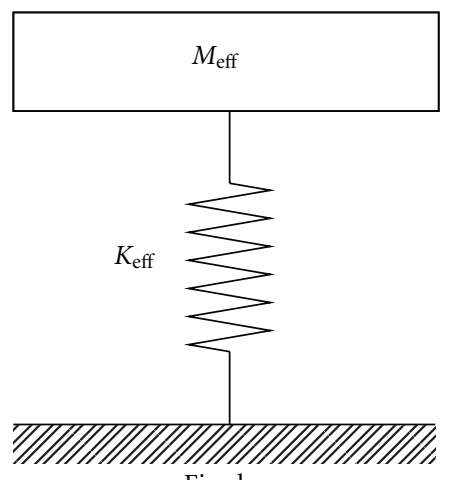

Fixed

(b)

Figure 1: (a) Schematic diagram and (b) mechanical lumped parameter model of piezoelectric microcantilever sensor (PEMS).

of its original area and that area is located at the end of the cantilever, the surface stress sensitivity is reduced to approximately one quarter of its original value. The change in surface stress that is induced by the attached biomolecules is typically in the range of $0.01-0.08 \mathrm{~N} / \mathrm{m}$ [6], and therefore, the change in the resonant frequency is calculated to be approximately in the range of $2.7-21.6 \mathrm{~Hz}$. The frequency changes due to surface stress are less than or equal to the frequency resolution of our PEMS.

The developed PEMS was fabricated using a standard micromachining technique, the details of which are described in our previous paper [14]. Figure 2(a) shows a cross-sectional diagram, and Figure 2(b) shows an optical image of the developed PEMS. The deep trench that can be seen in Figure 2(b) was created to improve the electrical properties of the PEMS.

\section{Experiments}

3.1. Materials. Recombinant HBsAg, monoclonal antiHBsAg, and alpha-fetoprotein (AFP) were purchased from HBI (South Korea). Phosphate-buffered saline (PBS, pH 7.4) and bovine serum albumin (BSA) were purchased from Affymetrix. Thiolated protein A/G (Protein A/G-SH) was prepared in the Magic Laboratory (POSTECH, South Korea) using Protein A/G (BioVision) and Traut's reagent (2iminothiolane hydrochloride) according to the instructions for the Traut's reagent (number 26101, Thermo Scientific). 
TABLE 1: Material properties and parameters used in mechanical lumped parameter model of piezoelectric microcantilever sensor (PEMS) [12].

\begin{tabular}{|c|c|c|c|c|}
\hline Quantity & Unit & Value & Expression & Remark \\
\hline \multicolumn{5}{|l|}{ Silicon } \\
\hline$E$ & $\mathrm{GPa}$ & 170 & Young's modulus & \\
\hline$\rho$ & $\mathrm{kg} / \mathrm{m}^{3}$ & 2329 & Density & \\
\hline$v$ & & 0.28 & Poisson's ratio & \\
\hline \multicolumn{5}{|l|}{ Piezoelectric layer } \\
\hline$E$ & $\mathrm{GPa}$ & Anisotropic & & PZT5H \\
\hline$\rho$ & $\mathrm{kg} / \mathrm{m}^{3}$ & 7500 & & \\
\hline Resonance frequency $\left(f_{0}\right)$ & $\mathrm{MHz}$ & 1.345479 & $\begin{array}{l}f_{0}=(1 / 2 \pi) \sqrt{K_{\text {eff }} / M_{\text {eff }}} \\
\text { In fundamental mode }\end{array}$ & Theoretical data \\
\hline Effective stiffness ( $\left.K_{\text {eff }}\right)$ & $\mathrm{N} / \mathrm{m}$ & 234 & $\begin{array}{l}K_{\text {eff }}=2 E_{\text {strain }} / \delta_{\max }^{2} \\
\text { where } E_{\text {strain }} \text { is strain energy and } \\
\delta_{\max } \text { is the maximum displacement } \\
\text { of the free end of the PEMS in } \\
\text { fundamental mode }\end{array}$ & Theoretical data \\
\hline Effective mass $\left(M_{\text {eff }}\right)$ & ng & 3.272 & $M_{\mathrm{eff}}=k_{\mathrm{eff}} / 4 \pi^{2} f_{0}^{2}$ & Theoretical data \\
\hline
\end{tabular}

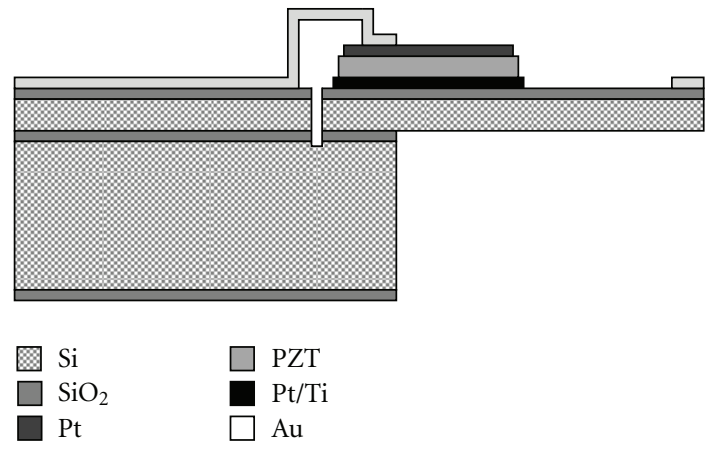

(a)

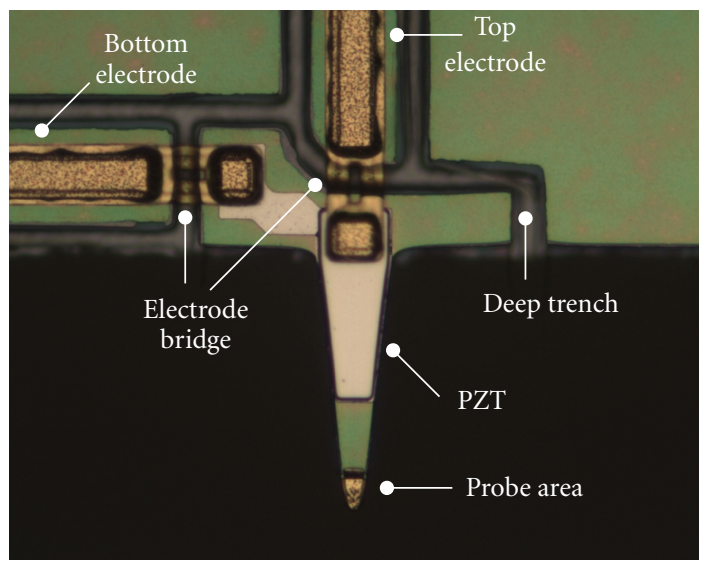

(b)

FIgURE 2: (a) Schematic cross-sectional diagram and (b) optical image of developed PEMS.

2-Propanol(isopropyl alcohol), ethanol, and methanol were purchased from Avantor Materials.

3.2. Experimental Setups. Figure 3 shows the experimental setups for the detection of HBsAg using the PEMS. The probe area of the PEMS was dipped into a droplet $(<10 \mu \mathrm{L})$ of biochemical solution, and the dipping depth was controlled using a linear stage for monitoring through a CCD camera. The resonant frequency of the PEMS was measured using a PXI system, which is a computer-based measurement device. The peak point of the conductance spectra of the PEMS is the mechanical resonant frequency [15], and the frequency at the peak point was calculated using a program developed using LabVIEW, a graphical program language. A thermohygrostat was used to maintain a constant humidity and temperature during the experiment.

3.3. Detection Procedure. The detection procedure used for HBsAg is shown in Figure 4, and its detailed steps are listed in Table 2. First, the sensor was subjected to a cleaning process that employed ultraviolet (UV) light (254 nm, UV5D Short-Wave Lamp, Spectronics), and wet cleaning was also applied. Second, the gold surface on the probe area was reacted with the thiolated protein $A / G$, which was used to bind the antibody. Third, the sensor was passivated with BSA to prevent nonspecific binding. Fourth, anti-HBsAg was immobilized on the reaction site of the sensor. Fifth, a control test using PBS and AFP was performed for comparison with the detection results of HBsAg. Finally, the HBsAg was detected at a specific concentration while controlling the reaction time. PBS rinsing and DI water rinsing were carried out at every step, and the resonant frequency of the PEMS was also measured at every step after drying with nitrogen $\left(\mathrm{N}_{2}\right)$ gas. The mass of the detected HBsAg was calculated using the difference in the resonant frequency before and after detection of the HBsAg and the mass sensitivity of the PEMS. In the process of immobilizing the anti-HBsAg, the concentration and reaction time at every step was determined experimentally, as listed in Table 2. The biochemical solution in the form of droplets was replaced several times to compensate for the effect of evaporation on the concentration. 


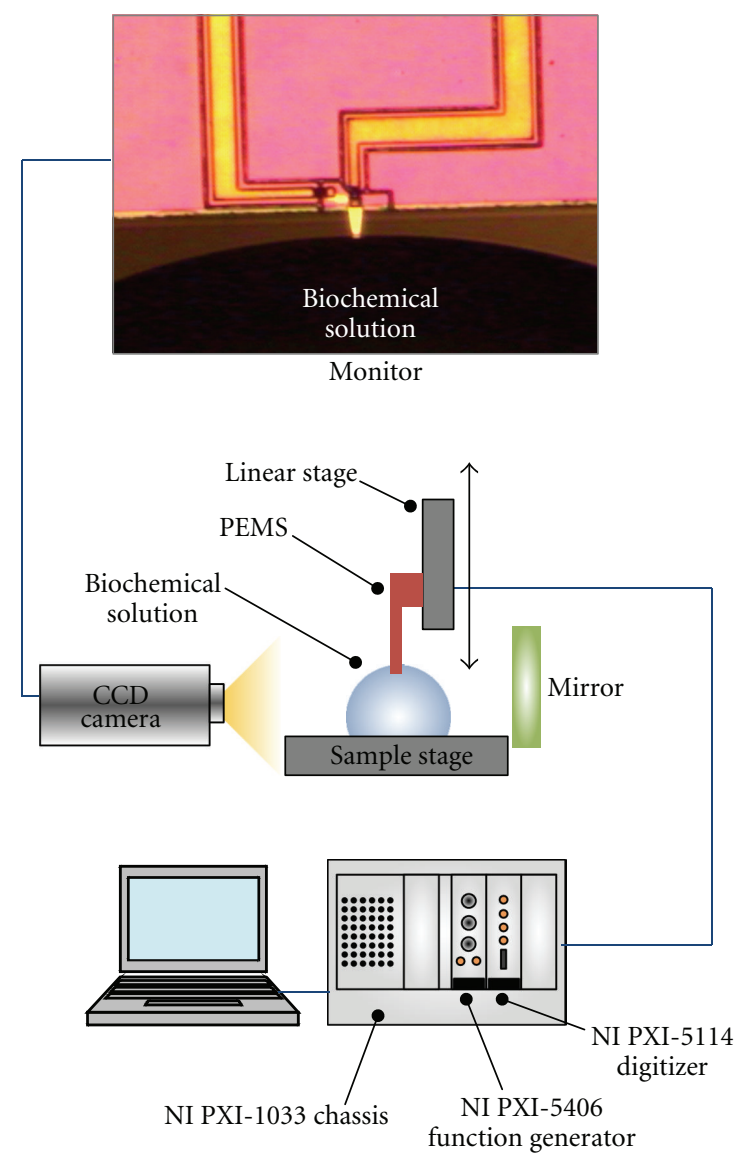

FIgure 3: Experimental setups for detection of HBsAg with PEMS using "dip- and-dry" technique.

\section{Results and Discussion}

The resonant frequency of the PEMS decreases after biomolecules are attached to it. Figure 5 shows the conductance spectra of the PEMS at every step in the process of immobilization of the anti-HBsAg on the probe area. The resonant frequency decreases progressively at each step. It takes less than $20 \mathrm{~s}$ to obtain a conductance spectrum, and the quality factor of the PEMS is approximately 200.

The mass amount of the detected HBsAg depends on the concentration of the target solution, the reaction time, the density of the immobilized anti-HBsAg, and the area it occupies on the probe. The reaction time with the target was maintained at a constant level for $10 \mathrm{~min}$. Furthermore, the anti-HBsAg density and its area on the probe were almost maintained at a constant level because the probe area was defined by identical patterned gold and employing the same processes used for immobilization of the antibody. In this way, the mass amount of the HBsAg depends only on the concentration; that is, the PEMS could measure the concentration of HBsAg in the target solution by measuring the mass of the detected HBsAg.

Figure 6 shows the mass amount of detected HBsAg in the range of $0.1-100 \mathrm{ng} / \mathrm{mL}$. As the concentration increases, the binding speed of the HBsAg also increases. Therefore, the mass amount of detected HBsAg during the reaction
TABLE 2: Procedures and time requirements in detection process of HBsAg.

\begin{tabular}{|c|c|c|}
\hline & Procedure & Time (min) \\
\hline \multirow{5}{*}{ Sensor cleaning } & UV cleaning & 30 \\
\hline & 2-Propanol cleaning & 10 \\
\hline & Ethanol cleaning & 30 \\
\hline & Methanol cleaning & 10 \\
\hline & DI water rinsing & 5 \\
\hline \multirow{4}{*}{ Protein A/G-SH } & Protein A/G-SH $(5 \mu \mathrm{g} / \mathrm{mL})$ & 40 \\
\hline & PBS rinsing & 5 \\
\hline & DI rinsing & 5 \\
\hline & $\mathrm{N}_{2}$ gas dry & 1 \\
\hline \multirow{4}{*}{ BSA } & BSA $(0.01 \%)$ & 30 \\
\hline & PBS rinsing & 5 \\
\hline & DI rinsing & 5 \\
\hline & $\mathrm{N}_{2}$ gas dry & 1 \\
\hline \multirow{4}{*}{ Anti-HBsAg } & Anti-HBsAg $(25 \mu \mathrm{g} / \mathrm{mL})$ & 30 \\
\hline & PBS rinsing & 5 \\
\hline & DI rinsing & 5 \\
\hline & $\mathrm{N}_{2}$ gas dry & 1 \\
\hline \multirow{7}{*}{ Control } & PBS & 10 \\
\hline & DI rinsing & 5 \\
\hline & $\mathrm{N}_{2}$ gas dry & 1 \\
\hline & AFP & 10 \\
\hline & PBS rinsing & 5 \\
\hline & DI rinsing & 5 \\
\hline & $\mathrm{N}_{2}$ gas dry & 1 \\
\hline \multirow{4}{*}{ HBsAg } & HBsAg & 10 \\
\hline & PBS rinsing & 5 \\
\hline & DI rinsing & 5 \\
\hline & $\mathrm{N}_{2}$ gas dry & 1 \\
\hline
\end{tabular}
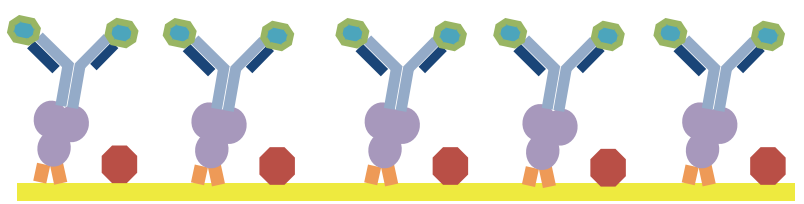

Probe area (gold)

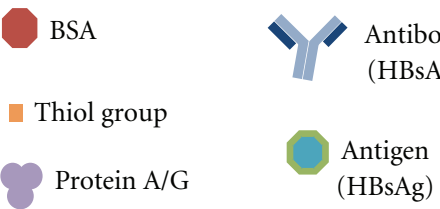

Figure 4: Procedure for detection of HBsAg with PEMS.

time of $10 \mathrm{~min}$ increased as expected. Figure 6 also shows the results of the measured mass that were obtained from control tests using PBS and AFP. The results for PBS are related to the minimum detectable mass, and the results for AFP are related to the binding selectivity of the detection test of HBsAg performed with the PEMS. 


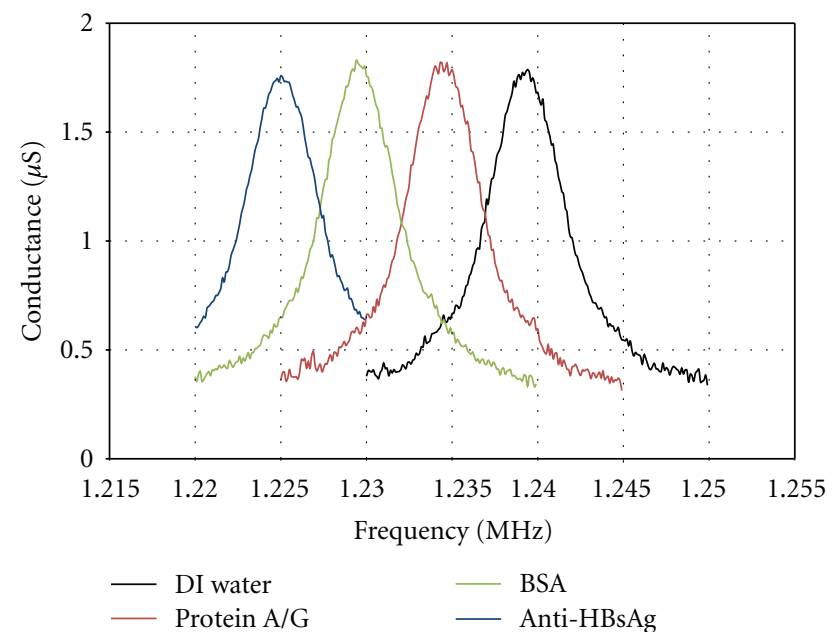

FIgure 5: Conductance spectra of PEMS in the process of immobilizing anti-HBsAg on probe area.

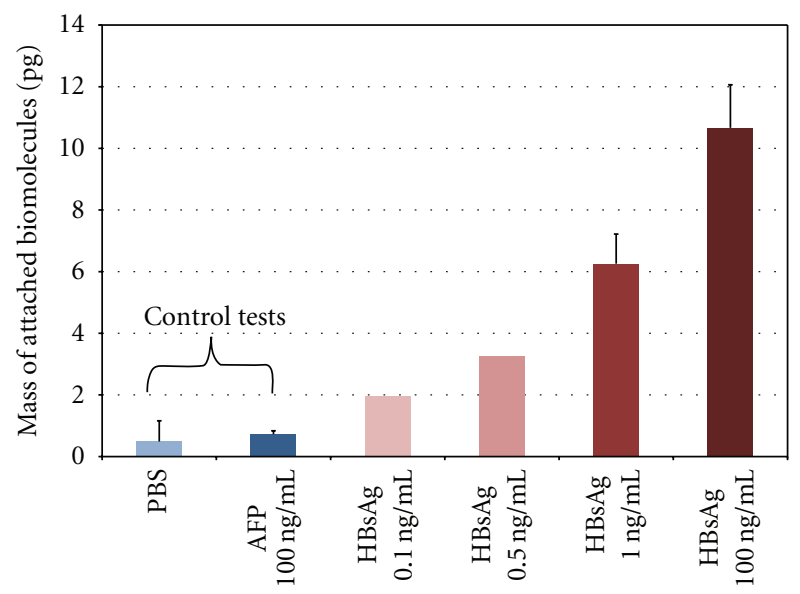

Figure 6: Mass of detected HBsAg in several concentrations.

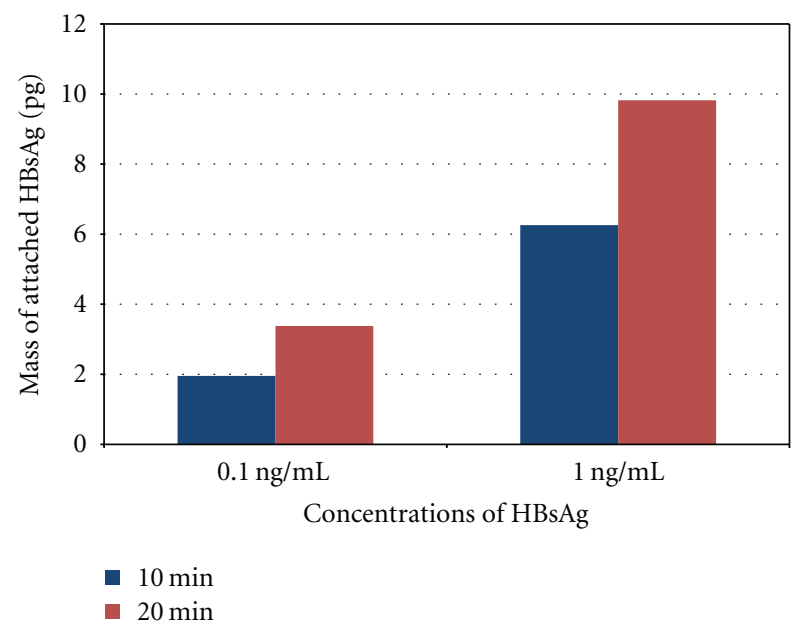

Figure 7: Time dependence of immunoreaction for different concentrations of HBsAg.
Figure 7 shows the time dependence of the immunoreaction for different concentrations. The mass increased by approximately $73 \%$ at a concentration of $0.1 \mathrm{ng} / \mathrm{mL}$ and $57 \%$ at a concentration of $1 \mathrm{ng} / \mathrm{mL}$, as the reaction time increased twofold. The binding speed of the HBsAg may decrease as the reaction time increases because the binding speed will be zero at the saturation point; therefore, the increasing ratios of the mass of detected HBsAg are less than $100 \%$. In addition, the increasing ratio has a lower value at a higher concentration of HBsAg because the binding speed of the HBsAg increases and then the binding reaction quickly reaches the saturation point. We need to maintain a constant reaction time while measuring the concentration of $\mathrm{HBsAg}$, but we need to adjust the reaction time as the detection region of the concentration. That is, a shorter reaction time is needed in the lower concentration region and a longer reaction time is needed in the higher concentration region.

In the immunoassay, flow injection analysis (FIA) is also widely used; it allows tracking the kinetics of the immunoreaction [9] and it is relatively useful for multiplexed detection or automated system. However, FIA needs more complicated systems compared to the analysis using "dipand-dry" technique. Furthermore, a PEMS operating in liquid has lower quality factor and lower resonant frequency due to viscous damping effect and added mass effect of the liquid $[4,7]$; thus, FIA could deteriorate the reliability and sensitivity of the PEMS. Therefore, "dip- and-dry" approach is appropriate for measuring the concentration of $\mathrm{HBsAg}$ with the developed PEMS.

\section{Conclusions}

In this paper, HBsAg concentrations were measured in the range of $0.1-100 \mathrm{ng} / \mathrm{mL}$ using a PEMS that was developed for use as a mass sensor. The "dip- and-dry" technique was used, and the concentration was measured by measuring the mass of the detected HBsAg while keeping the reaction time for the target solution, the anti-HBsAg density, and its area on the probe constant. From the results obtained, we expect that the piezoelectric microcantilever mass sensor can be utilized for the measurement of the concentration of HBsAg and can also be used for sensitive diagnostic testing for HBV infection.

\section{Acknowledgments}

This work was supported by the "Development of Bio Robot Technology for POCT (point-of-care testing) (10024720)" program under the Industrial Source Technology Development Programs of the Ministry of Knowledge Economy (MKE) of Korea and was partly supported by a National Research Foundation of Korea (NRF) grant funded by the Korea government (MEST) (no. 2010-0019292).

\section{References}

[1] S. P. S. Monga, Ed., Molecular Pathology of Liver Diseases (Molecular Pathology Library 5), Springer, New York, NY, USA, 2011. 
[2] S. C. Lou, S. K. Pearce, T. X. Lukaszewska, R. E. Taylor, G. T. Williams, and T. P. Leary, "An improved Abbott ARCHITECT ${ }^{\circledR}$ assay for the detection of hepatitis B virus surface antigen (HBsAg)," Journal of Clinical Virology, vol. 51, no. 1, pp. 59-63, 2011.

[3] K. S. Hwang, S. M. Lee, S. K. Kim, J. H. Lee, and T. S. Kim, "Micro- and nanocantilever devices and systems for biomolecule detection," Annual Review of Analytical Chemistry, vol. 2, pp. 77-98, 2009.

[4] S. Xu and R. Mutharasan, "Cantilever biosensors in drug discovery," Expert Opinion on Drug Discovery, vol. 4, no. 12, pp. 1237-1251, 2009.

[5] Q. Ren and Y. P. Zhao, "Influence of surface stress on frequency of microcantilever-based biosensors," Microsystem Technologies, vol. 10, no. 4, pp. 307-314, 2004.

[6] S. Shin, J. P. Kim, S. J. Sim, and J. Lee, "A multisized piezoelectric microcantilever biosensor array for the quantitative analysis of mass and surface stress," Applied Physics Letters, vol. 93, no. 10, Article ID 102902, 2008.

[7] Y. Lee, G. Lim, and W. Moon, "A piezoelectric micro-cantilever bio-sensor using the mass-micro-balancing technique with self-excitation," Microsystem Technologies, vol. 13, no. 5-6, pp. 563-567, 2007.

[8] D. W. Chun, K. S. Hwang, K. Eom et al., "Detection of the $\mathrm{Au}$ thin-layer in the $\mathrm{Hz}$ per picogram regime based on the microcantilevers," Sensors and Actuators A: Physical, vol. 135, no. 2, pp. 857-862, 2007.

[9] L. Nicu, M. Guirardel, F. Chambosse et al., "Resonating piezoelectric membranes for microelectromechanically based bioassay: detection of streptavidin-gold nanoparticles interaction with biotinylated DNA," Sensors and Actuators B: Chemical, vol. 110, no. 1, pp. 125-136, 2005.

[10] T. Kwon, K. Eom, J. Park, D. S. Yoon, H. L. Lee, and T. S. Kim, "Micromechanical observation of the kinetics of biomolecular interactions," Applied Physics Letters, vol. 93, no. 17, Article ID 173901, 2008.

[11] Y. Lee, Mass detection technique using piezoelectric micro cantilever and its application to bio sensor, Ph.D. thesis, Pohang University of Science and Technology, Pohang, Republic of Korea, 2008.

[12] S. Lee et al., "Improvements in electrical properties of piezoelectric microcantilever sensors by reducing parasitic effects," Journal of Micromechanics and Microengineering, vol. 21, Article ID 085015, 2011.

[13] D. Lee, M. Yoo, H. Seo et al., "Enhanced mass sensitivity of $\mathrm{ZnO}$ nanorod-grown quartz crystal microbalances," Sensors and Actuators B: Chemical, vol. 135, no. 2, pp. 444-448, 2009.

[14] Y. Lee, S. Lee, H. Seo, S. Jeon, and W. Moon, "Label-free detection of a biomarker with piezoelectric micro cantilever based on mass micro balancing," Journal of the Association for Laboratory Automation, vol. 13, no. 5, pp. 259-264, 2008.

[15] IEEE Standard, "177-1966-IEEE Standard Definitions and Methods of Measurements for Piezoelectric Vibrators," 1966. 

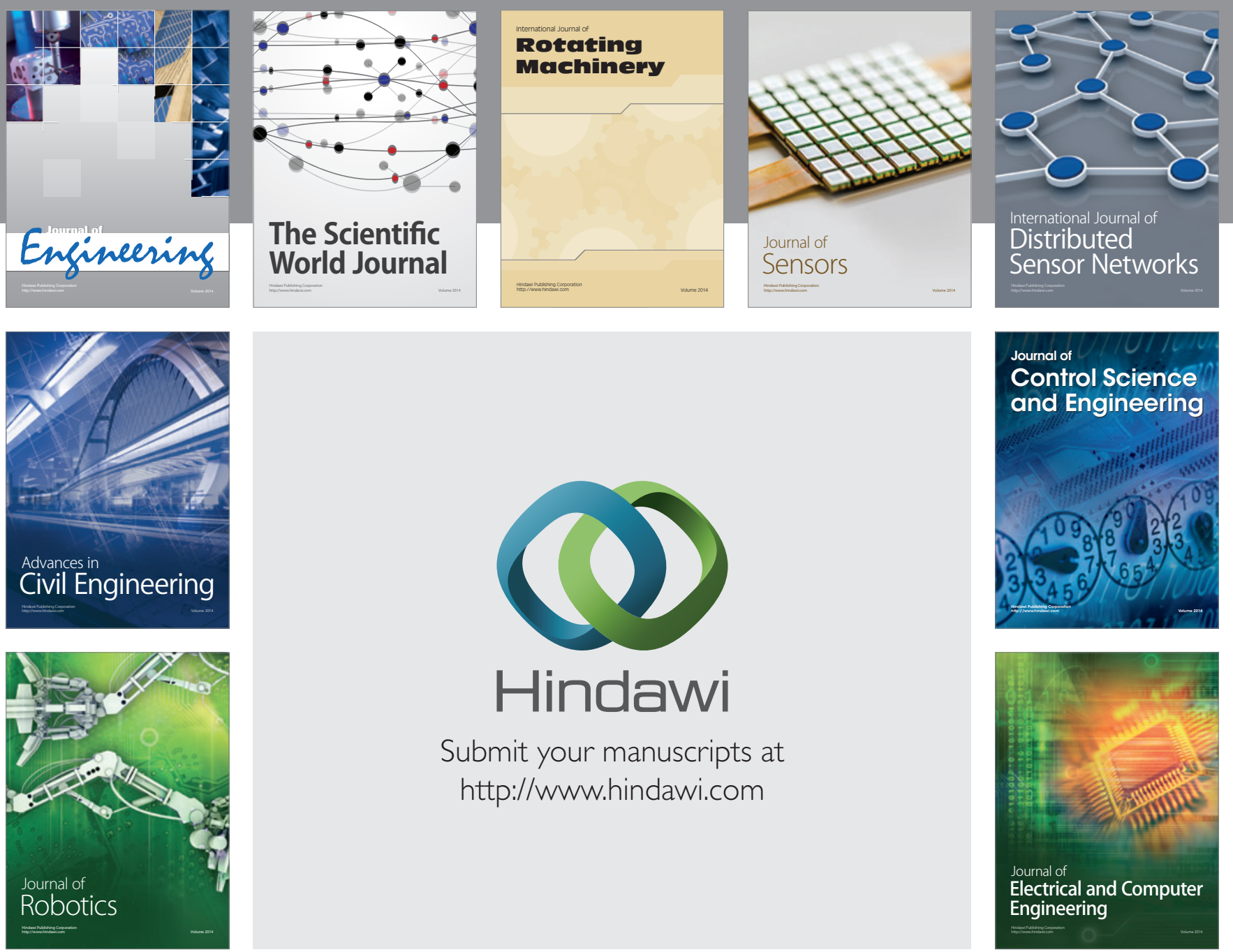

Submit your manuscripts at

http://www.hindawi.com
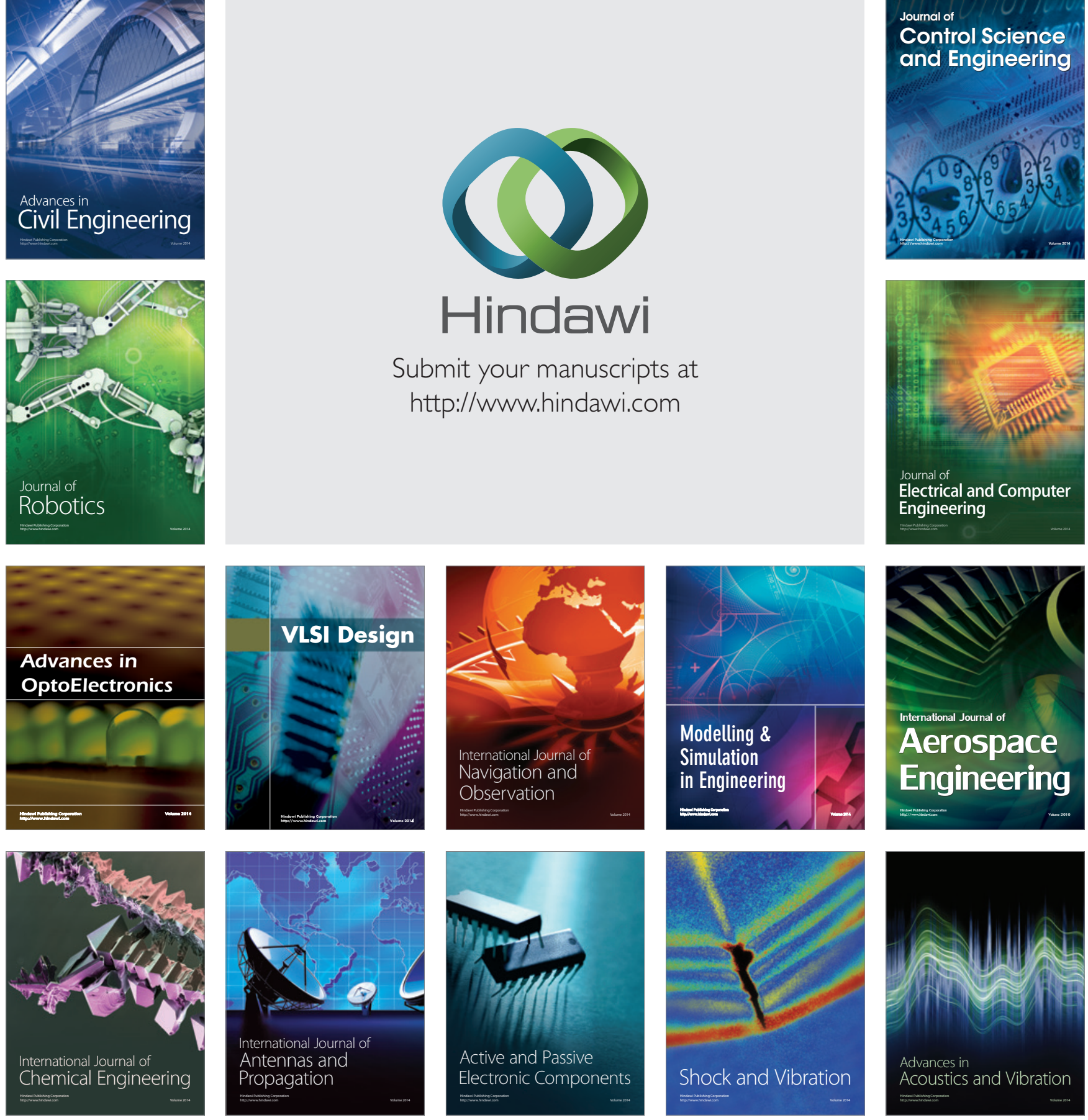